\title{
MODELO DE EVALUACIÓN DE LA CAPACIDAD DE ACOGIDA DEL TERRITORIO CON SIG Y TÉCNICAS DE DECISIÓN MULTICRITERIO RESPECTO A LA IMPLANTACIÓN DE EDIFICACIONES EN ESPACIOS RURALES
}

\author{
Federico Benjamín Galacho Jiménez y Juan Antonio Arrebola Castaño \\ Departamento de Geografía, Universidad de Málaga
}

\section{RESUMEN}

Se muestra un método, que apoyado en propuestas científicas, ayuda en la planificación del uso sostenible del territorio. Dicho método se basa en los modelos de evaluación de la capacidad de acogida del territorio. Su fundamento es el desarrollo de una metodología de evaluación con una perspectiva amplia de la sostenibilidad rural y contempla la necesaria puesta en valor de los recursos naturales, la preservación del medio por su calidad ambiental y la minimización de los riesgos naturales. Con él se evaluará la implantación de edificaciones relacionadas con el proceso de urbanización difusa en los espacios rurales. Para ello se aborda la evaluación de las condiciones particulares del medio rural en orden a la mejor estimación de sus posibilidades y de su vulnerabilidad respecto a los riesgos naturales. Como resultado se ha realizado una evaluación basada en juicios de valor claramente definidos y en atributos concretos del territorio. Mediante este proceso se obtiene una capa de información que muestra una clasificación del espacio estudiado con una valoración asignada a cada parte del territorio en función de su capacidad para acoger los usos que han sido evaluados.

Palabras claves: sistemas de información geográfica, técnicas de decisión multicriterio, capacidad de acogida, medio rural.

\section{ABSTRACT \\ Evaluation model of land carrying capacity with GIS and decision techniques multicriteria regarding the implementation of building in rural areas}

It shows a method that relied on scientific proposals, assistance in planning sustainable land use. This method is based on evaluation models of carrying capacity of the land. Its foundation is the development of an evaluation methodology with a broad perspective of rural sustainability and provides the necessary enhancement of natural resources, the preservation of their environmental quality and the minimization of natural hazards. With it will assess the implementation of process-related buildings urban sprawl in rural areas. This evaluation addresses the particular conditions of rural spaces in order to better estimate their potential and their vulnerability to natural hazards. As a result, the evaluation was based on value judgments based on clearly defined and specific attributes of the territory. This process results in a layer of information that shows a classification of the studied space with a score assigned to each part of the territory according to its ability to host applications that have been evaluated.

Key words: geographic information systems, multicriteria decision thecniques, capacity models, rural spaces.

\section{INTRODUCCIÓN}

Se parte de la consideración de que el conocimiento de las formas de ocupación, los nuevos usos o funciones y las consecuencias derivadas de ello en aquellas partes del territorio que no son consideradas como urbanas se revela esencial para poder abordar con ciertas garantías la ordenación y planificación integral del territorio. Mientras que en los espacios urbanos la regulación edificatoria y urbanizadora,

Contacto: Federico Benjamín Galacho Jiménez: fbgalacho@uma.es; Juan Antonio Arrebola Castaño: juan.arrebola@uma.es 
incluso su incidencia en el entorno, se encuentra claramente definida y estructurada, en los espacios rurales los modelos de crecimiento, de colonización del espacio, de renovación de la edificación tradicional se desarrollan de modo incontrolado. Valga como muestra observar como se han extendido y vulgarizado los tipos de construcciones de casas de campo que se han convertido en símbolos regionalistas o en la base de un repertorio estilístico o adulterado de "chalecitos" característicos de cualquier urbanización en el medio urbano que se precie (recuérdese sin ir más lejos cualquier anuncio inmobiliario pregonando las excelencias de la casa tipo cortijo, masía, caserío, villa mediterránea o incluso más exóticos como el tirolés, ibicenco, provenzal, alpino, etc.). En este terreno, en el que no vamos a entrar, se ha llegado a límites caricaturescos, con nefasta incidencia territorial. La diferencia entre los tipos arquitectónicos urbanos y los rurales no es otra que de escala. Por eso, lo que nos interesa aquí es analizar la capacidad del proceso constructivo de configurar el paisaje y de ordenarlo (o desordenarlo, según se mire), además de la incidencia de éste en el medio rural. Por consiguiente, para observar lo que nos interesa analizar, es necesario un alejamiento físico e intelectual que permita ver, no los componentes o la estructura orgánica del edificio o la implantación, con ser éstos de sumo interés, sino su área de influencia, su incidencia en el paisaje, su capacidad ordenadora, su papel humanizador o su impacto ambiental. Estas consideraciones surgen de una constatación de la realidad analizada. En el medio rural ya existía un modelo de hábitat, en cada lugar prevalecía un tipo arquitectónico propio, adaptado a las características del terreno y a su paisaje.

Paralelamente se abren las posibilidades para su planificación, especialmente si se trata de armonizar desarrollo y sostenibilidad. Sin embargo, la extensión del proceso urbano a estos espacios resulta especialmente grave. Fundamentalmente por cuatro aspectos: por la forma como se ha venido desarrollando: sobre una base especulativa, porque sus efectos se han visto acentuados al desarrollarse sobre espacios de funcionalidad rural predominante que adolecen de una fragilidad y vulnerabilidad característica, porque el modelo se fundamenta en una fuerte demanda residencial de élites socio-económicas de orígenes diversos y, por la dejadez de la responsabilidad política de las distintas administraciones en sus competencias de actuación sobre ellos. En Galacho (2010 y 2011) se vierten algunas reflexiones al respecto.

En esta situación nos ha parecido de utilidad diseñar un instrumento de apoyo a la decisión, que fundamentado en propuestas científicas, ayude en el marco amplio de la planificación del uso sostenible del espacio rural. Para ello se considera como primordial abordar la ordenación de los usos edificatorios en el medio rural a través de una evaluación de la aptitud del territorio para acogerlos y de sus efectos inducidos con especial referencia a los riesgos naturales. Así evaluar las condiciones particulares del medio rural en orden a la mejor estimación de sus posibilidades, realizar evaluaciones basadas en juicios de valor claramente definidos y basándose en atributos concretos del territorio para desarrollar simulaciones y establecer escenarios de actuaciones distintos. Con estas finalidades se acomete, como eje fundamental, el desarrollo de esta metodología de evaluación con una perspectiva amplia de la sostenibilidad rural: contemplando la necesaria puesta en valor de los recursos naturales, la utilización del medio en proporción a su calidad ambiental y su sostenibilidad social.

La propuesta metodológica que hacemos se inserta en el marco de la investigación en planificación física y en el desarrollo rural, y en el ámbito instrumental de los Sistemas de Información Geográfica (SIG) y las Técnicas de Evaluación Multicriterio (EMC).

El concepto de capacidad de acogida es considerado como un concepto teórico que hace referencia al uso óptimo del territorio en orden a su sostenibilidad. Más concretamente, Gómez Orea $(1992,25)$ lo define como "el grado de idoneidad o cabida que presenta el territorio para una actividad teniendo en cuenta a la vez, la medida en que el medio cubre sus requisitos locacionales y los efectos de dicha actividad sobre el medio". De ello se deriva que los usos evaluados obtendrán su localización óptima cuando sean asignados en un lugar que los pueda recibir sin que se degraden gravemente sus características ecológicas o paisajísticas, de tal manera que su integración en el medio cuente con la mayor aptitud y el menor impacto posibles. Por tanto las propiedades del territorio son valoradas en su significado con relación al desarrollo de las actuaciones. Consideradas dichas propiedades en su conjunto, para cada espacio determinado y para unas posibles actuaciones concretas. En este contexto, el significado de capacidad de acogida del territorio se entiende derivado de la concurrencia de ciertas características y elementos ambientales significativos en un espacio determinado. 
Dicho concepto fue puesto en práctica, en un principio, dentro de la teoría de la planificación física con base ecológica que se ha venido desenvolviendo con el soporte de diferentes propuestas metodológicas basadas en procedimientos y criterios de clasificación, agregación y análisis. Cabe destacar en esta línea las aportaciones sucesivas de: Canter (1977, 1979 y 1985), Clark (1978, 1980), Bisset (1980), Rau y Wooten (1980), Hollick (1981), Lee (1982 y 1983) y Black (1991), entre otros.

En la práctica de la ordenación territorial y el planeamiento urbanístico este concepto teórico se ha desarrollándo sobre dos basamentos: el análisis de las aptitudes y el análisis de los impactos. El primero, que se orienta a la valoración de las oportunidades que el medio ofrece al desenvolvimiento de la actividad humana, es una práctica básica en la planificación territorial. El segundo, el análisis de los impactos, cuya base son las directrices de protección, parte de la valoración de la fragilidad del medio, a fin de establecer las limitaciones de uso que puedan impedir su deterioro. La integración de estas dos líneas de evaluación del territorio, la de aptitud y la de impacto, puede derivar en la elaboración de un modelo territorial ideal, en el que se optimice el aprovechamiento de los recursos y la implantación de las actividades. Este modelo se basa en los mismos conceptos de aptitud (que resume el grado de adaptación del medio a los requerimientos del objeto para el que es evaluado) e impacto (los efectos negativos que pueden derivarse de la implantación de las actividades). Se incluye también de modo habitual el concepto de restricción, al desarrollarse la metodología a modo de evaluación orientada a un objetivo preciso: delimitar entre las alternativas reales las que son incompatibles (natural o normativamente) con el objeto de la evaluación.

En Galacho y Ocaña (2006) realizábamos una propuesta metodológica basada en un modelo de evaluación que pretendía medir la capacidad del territorio para acoger los usos comúnmente previstos en los planes de ordenación urbanística; en ese caso, residenciales y comerciales en nuevos sectores de planeamiento. Se diseñaba y aplicaba un método que fundamentado en información territorial y urbanística, organizada y actualizada, servía para la obtención de valores de capacidad de acogida del territorio para cada uso o actividad propuesto en los documentos de planeamiento urbanístico. Con todo ello se pretendía que la información espacial generada se pudiese utilizar de apoyo a la toma de decisiones en las distintas fases de redacción de los documentos de planeamiento y que ésta se realizase sobre una base objetiva que permitiese evaluar los procesos que determinan la realidad urbana y territorial de los municipios; y sobre todo, para el diseño de las implantaciones de los suelos urbanizables que darían lugar a los desarrollos urbanísticos futuros. Posteriormente, en las sucesivas aplicaciones del modelo diseñado entonces observamos una serie de dificultades inherentes a los procesos de planificación derivados esencialmente de un hecho: la dificultad de cuantificar en su justa medida los efectos territoriales de las actividades económicas en general y de los usos urbanísticos concretos en particular. Con ello queremos decir que un método como el propuesto puede conducir a distintas posibilidades o soluciones, representadas gráficamente en mapas con diferentes grados de capacidad de acogida.

La metodología que ahora proponemos, evolucionada de la anteriormente mencionada, ha sido objeto de una profunda revisión. De acuerdo al nuevo objeto de evaluación, concerniente a la consideración de los efectos inducidos por la implantación de edificaciones aisladas en espacios rurales, se plantea ahora que sobre el escenario actual se genere una zonificación que abarque todo el territorio y sirva para crear un marco de referencia que regule los crecimientos o implantaciones futuras. En dicho escenario, que sirve de punto de partida espacio-temporal, las actuaciones existentes pueden ser valoradas como negativas o como positivas. Bajo este supuesto se pueden establecer estrategias distintas. Se puede optar por maximizar o minimizar los efectos de las implantaciones según distintos argumentos y circunstancias. Si optamos por maximizar los efectos negativos y minimizar los positivos, el resultado será el particular a este enfoque. Y en este sentido, se contrapone los conceptos de fragilidad, singularidad, rareza, etc., a otras consideraciones de sociales y económicas. Obtendremos otro resultado si actuamos de modo contrario, y el enfoque se centra en esgrimir los efectos positivos sobre los negativos que pueden encontrarse en el área objeto de aplicación, donde, a su vez, pueden darse entrada a gran número de alternativas razonables relacionadas con este objetivo. De cualquier manera consideramos que la valoración del óptimo debe evidenciar la adecuación del área de aplicación del modelo a determinados usos y también a diferentes grados de conflicto. Así, la aptitud óptima realza la capacidad territorial para acoger las actuaciones, con matices derivados de las posibles orientaciones favorables que puedan inducirse sobre los elementos espaciales y los procesos actuantes por la implantación de dichas actividades. 
Por consiguiente, la lógica del modelo de evaluación no se construye sobre un proceso cerrado, cuyo inicio es la formulación del objeto de la valoración y su fin la obtención de una propuesta final única, sino a través de posibles alternativas y según diversos escenarios. Aunque en la práctica esto complica enormemente el diseño del modelo, no obstante, con ello se avanza en la construcción de escenarios futuros, y el método puede dotarse de un cariz predictivo. Pero para ello, metodológicamente, necesitamos llegar a valoraciones con una desagregación muy elevada de juicios parciales.

La utilización combinada de los Sistemas de Información Geográfica (SIG) y las Técnicas de Evaluación Multicriterio (EMC) se constituyen en unas herramientas muy útiles para su aplicación en los procesos de evaluación territorial. Así el método adquiere la máxima aplicabilidad posible, siendo posible investigar el número de alternativas y, en definitiva, facilitar la toma de decisiones. Como se afirma, las ventajas de utilizar estas técnicas con SIG se sitúan en poder resolver con todo rigor la interrelación de las diversas variables del territorio, otorgándosele el peso y la proporcionalidad adecuada o necesaria en cada momento. Un atributo cualquiera contenido en cada una de las capas de información de un SIG, pueden ser dentro de él, ponderado como elemento positivo o negativo según un determinado objeto. Y puede igualmente ser valorado en conjunción con otros y en función de ello contrarrestado, potenciado o anulado. En base a esto, un aspecto crucial de la metodología es el diseño de criterios y factores que fundamentan las distintas evaluaciones, así como la necesaria experimentación en un ámbito geográfico determinado. A su vez, en lo que concierne al proceso de evaluación, su diseño dentro de un SIG, permite aprovechar las potencialidades de éste en la interrelación de las capas de información requeridas (capas que darán lugar a criterios y factores) y sus utilidades de análisis/evaluación; de modo que sea factible su aplicación y revisión en otros escenarios espaciales con la mera adecuación de los datos, y en su caso de los procesos, a las nuevas condiciones geográficas o territoriales. Con ello el método adquiere un carácter aplicado y una finalidad demostrativa y se convierte en una herramienta dúctil, con la que se puede interactuar, capaz de reorientar las evaluaciones de acuerdo a diferentes puntos de vista. Así los resultados serán validos en función de los juicios y las valoraciones emitidas, teniendo cabida por tanto experimentar sobre resultados alternativos, rectificar los juicios, considerar o no determinadas variables o criterios, etc.

Han proporcionado fundamentos científicos a estos procedimientos y técnicas los siguientes autores: Nijkamp (1977, 1990), Voogd (1983), Seo (1988), Janssens (1992), Eastman et al., (1993a,b), Jankowski (1995), Gal et al., (1999), Malczewski (1999), Triantaphyllou (2000), Belton y Stewart (2002), Roy (2007) y Munda (2008); y en España, Romero (1993), Barredo (1996), Barba y Pomerol (1997), Santos $(1997,1998)$ y Moreno $(2000,2002)$. La utilidad de estos procedimientos es reconocida y el campo de aplicación de la EMC combinada con SIG para la evaluación de la capacidad de acogida del territorio en diversas temáticas es extenso, citamos algunos trabajos: Barredo y Bosque (1995), Ocaña y Galacho (2002), Galacho et al., (2004), Bosque y Moreno (2004), Gómez y Barredo (2005), Molero et al., (2007), Moreno y Buzai (2008) y Galacho et al., (2009).

\section{PROPUESTA METODOLÓGICA}

\subsection{Planteamientos de partida}

Nuestro objetivo principal será obtener una capa de información que muestre una clasificación del espacio estudiado con una valoración asignada a cada parte del territorio en función de su capacidad para acoger los usos que son evaluados: en este caso, las edificaciones y construcciones según distintas tipologías. Posteriormente con esta capa se procederá a asignar la valoración obtenida a dichas edificaciones.

Con la información resultante se podrá proceder a la generación de alternativas, y se podrá determinar en qué zonas se acoge más adecuadamente el uso analizado o las tipologías que presenta dicho uso. $\mathrm{O}$ en este caso, como podemos encontrar varias tipologías a evaluar: edificaciones aisladas, bien de uso residencial o no (naves, almacenes, etc.), sectores edificatorios de baja densidad, concentración de edificaciones aisladas que por su proximidad parcelaria dan lugar a hábitat concentrado, etc..., cuál o cuáles de ellas se adecuan mejor en cada punto. 
Por consiguiente, en el marco de esta propuesta metodológica la definición del objeto de la evaluación es un aspecto fundamental porque va a determinar la secuencia de la evaluación multicriterio, entendida ésta como un proceso lógico. Concretamente de ello dependerá la definición del proceso de evaluación posterior, y fundamentalmente la selección de los variables territoriales que formarán parte de la evaluación, la configuración de los factores y criterios y sus puntuaciones.

\subsection{Desarrollo del proceso metodológico}

\subsubsection{Definición del objeto y criterios de la evaluación}

Partimos de que centraremos nuestro objeto en la evaluación de la capacidad de acogida del territorio (espacios rurales en este caso) para implantación de edificaciones e instalaciones según las diversas tipologías que se pueden dar.

El objeto de evaluación establecido determina la definición de los criterios de la evaluación. Esta es una cuestión básica que se aborda con una finalidad operativa y determina como dichos criterios serán caracterizados y medidos; de ello dependerá gran parte del resultado final del proceso de evaluación. Esta propuesta metodológica de un solo objetivo define dos criterios principales: aptitud y vulnerabilidad al riesgo natural.

El primer criterio que se aborda es el de aptitud. Este criterio hace referencia a las cualidades del territorio, potenciales y/o restrictivas, para la implantación de las instalaciones y edificaciones determinadas en el objeto de la evaluación. Para ello el método se dirige a valorar tres aspectos del medio físico que determinarán las cualidades del territorio para el objeto propuesto y que se configuran en base a tres criterios: la aptitud física para la construcción, la aptitud ambiental y la capacidad de absorción visual.

En el denominado criterio de aptitud física para la construcción se consideran las condiciones constructivas de los terrenos. Con ello se pretende valorar las características del terreno en función de su idoneidad para la edificación y sus condicionantes técnicos. Se valoran dentro del criterio de aptitud física para la construcción: las variables pendiente y litología según sus cualidades de compresibilidad y capacidad portante y el factor estabilidad del terreno, que combina los grados de la pendiente con la resistencia de los tipos litológicos (tendencia al desplazamiento).

Para el denominado criterio de aptitud ambiental se ha considerado el análisis de los factores ambientales que pueden ser susceptibles a la degradación, considerando el suelo respecto a su capacidad agrológica y a su vocación forestal, y las características de la vegetación natural. La valoración de los suelos se aborda desde dos perspectivas: una, según su capacidad agrológica, considerada como recurso para la actividad agrícola más que por sus características naturales; y dos, en función de la valoración de sus condiciones de comportamiento forestal. De acuerdo a esto, la capacidad agrológica de los suelos es un elemento de caracterización fundamental para la definición de las unidades básicas del entorno rural por cuanto de ello derivan los usos y aprovechamientos agrícolas y no agrícolas. Por su parte, la vegetación natural se trata fundamentalmente considerando las zonas de vegetación natural, basándonos en la estructura, fisonomía y especies dominantes. No obstante, se da el caso de que en muchas zonas la vegetación natural sea bastante relicta, y entonces se puede optar por considerar entonces la vegetación potencial.

Como tercer criterio de aptitud hemos aludido al criterio de capacidad de absorción visual donde se valora la aptitud que tiene un paisaje de absorber visualmente modificaciones o alteraciones sin detrimento de su calidad visual. Se opta por abordar el tratamiento del paisaje considerando solamente sus cualidades visuales y prescindiendo del resto de percepciones sensoriales, con la finalidad de poder valorar la integración de las edificaciones o implantaciones. El objeto de este criterio es determinar las áreas visibles desde un conjunto de puntos relevantes y en base a ello se establece la medida en qué cada área contribuye a la percepción del paisaje y a la obtención de parámetros globales que permiten caracterizar el territorio objeto de estudio en términos visuales. En este sentido podemos destacar el ensayo de evaluación del paisaje visual mediante SIG realizado por Ocaña et al., (2004). Así pues, las condiciones de visibilidad son consideradas como cualidades del paisaje, lo que lleva a matizar, que mientras para el resto de los 
criterios, la calidad es un concepto relativo a la capacidad de acoger una determinada actividad, para el paisaje es un término absoluto, por lo que dichas cualidades son indicadores de la calidad del medio.

El segundo criterio mencionado como principal es el de la vulnerabilidad al riesgo natural. Se parte del hecho constatado que en numerosos casos la implantación de las edificaciones, instalaciones, etc. se realiza en lugares de riesgo y a su vez, las acciones relacionadas con su localización pueden modificar el entorno y generar riesgos inducidos, los cuales no sólo se pueden dar en el espacio inmediato de localización sino también pueden llegar a sentirse a distintas considerables de donde tuvieron su origen (por ejemplo, cuando se trata de modificar los cauces en su cabeceras o alterar la cubierta vegetal). En la configuración de este criterio interesa pues considerar la vulnerabilidad potencial a los riesgos y al mismo tiempo, los riesgos inducidos. En este sentido, se evidencia por tanto que no sólo se debe considerar el riesgo en sí mismo sino también es adecuado hacer depender la asignación de pesos de la valoración de las características de las edificaciones o construcciones a implantar. Pero es habitual que se de el caso de que no se disponga de un dimensionamiento efectivo de dichas edificaciones, y es cosa frecuente que sólo se puedan valorar propuestas de usos o instalaciones, por lo que es recomendable optar por medir el riesgo potencial de acuerdo a las características del medio. Por ello como mínimo es imprescindible localizar las actuaciones propuestas.

El criterio de vulnerabilidad al riesgo natural se conforma con cuatro factores: alteración morfodinámica, movimientos gravitacionales, inundación e incendios forestales.

La alteración morfodinámica hace referencia fundamentalmente a la erosión, en el sentido de que bajo esta denominación se engloban un conjunto de procesos físicos y químicos que producen modificaciones de las formas superficiales. Entendemos que la implantación de edificaciones, o asimismo las actuaciones relacionadas con ellas como movimientos de tierras para el allanamiento del terreno, viales de acceso, etc. puede derivar en la activación de procesos, latentes o no, de erosión, bien por aceleración de procesos activos, bien por alteración de las condiciones funcionales del terreno. Al mismo tiempo, los cambios de usos del suelo derivados de la implantación de edificaciones pueden ser altamente perturbadores de los fenómenos erosivos, generando riesgos inducidos para la población en combinación con otros factores (activación de los movimientos en masa y alteración del funcionamiento de la red hídrica superficial).

Respecto a los movimientos gravitacionales hay que decir que en cuanto la topografía deja de ser llana es relevante otorgar importancia a los riesgos asociados a procesos gravitacionales. Como se sabe, normalmente estos fenómenos se producen por la confluencia de un conjunto de factores relacionados con las características fisiográficas de los terrenos: pendiente, litología, cobertura vegetal, profundidad del suelo y desarrollo edáfico, fracturación y meteorización de la roca, orientación de las laderas, etc. En la configuración de este criterio se identifican los diferentes tipos de riesgo asociados con cada uno de los tipos de movimientos, que pueden ser caídas de bloques, deslizamientos, flujos y movimientos complejos. Comúnmente los procesos gravitacionales se suceden de modo asociado, una asociación característica es la que se produce entre deslizamientos y flujos. Así, esta conjunción y la magnitud en que se produce o se pueda producir determinan la proporcionalidad del riesgo y por tanto, las puntuaciones que se derivan en el proceso de evaluación.

La vulnerabilidad del terreno frente al riesgo de inundación se resuelve mediante la elaboración de las áreas inundables para los periodos de retorno definidos por las directrices de Protección Civil: 50, 100 y 500 años. Para ello se ha utilizado el clásico método hidrometeorológico con las fórmulas de Témez a partir del cual se determinan los caudales de referencia para un determinado periodo de retorno. Este método hidrometeorológico es el recogido en el BOE $n^{\circ} 123$ (instrucción de carreteras 5.2-IC «drenaje superficial»). En él, quedan recogidos los criterios y fórmulas que hemos tenido en cuenta en el cálculo de los caudales de avenida.

Por último, se ha considerado también el riesgo de incendios forestales. La magnitud y frecuencia de los episodios acaecidos en España durante los periodos estivales hacen que la consideración de la peligrosidad asociada a los incendios forestales esté justificada en este método. Aún más si tenemos en cuenta que el espacio rural ha venido siendo ocupado durante los últimos años por un urbanismo difuso que complica en gran medida cualquier tarea de extinción y obliga a fuertes medidas de prevención. Este criterio se configura en función de tres elementos que influyen principalmente en un incendio: el meteorológico, el topográfico y el factor combustibilidad. 
Finalmente, se ha establecido un filtro de restricciones compuesto por dos grupos de criterios: restricción legal e imposibilidad física. El primero hace referencia a las restricciones legales impuestas por las distintas normativas y legislaciones sectoriales vigentes: dominios públicos, protecciones ambientales, suelos protegidos, etc. y el segundo a la imposibilidad física que impida realizarse cualquier iniciativa constructiva, bien por la presencia de láminas de agua, o bien por cualquier otra circunstancia similar. Cada uno de ellos se convierte en claro criterio de restricción, de tal manera que cada criterio por sí mismo puede calificar zonas diferentes del territorio, de modo que un área puede verse afectada por sólo uno de ellos o por los dos. No es pertinente establecer valoración alguna, respecto a la importancia relativa de uno u otro, pues como se ha dicho, cada uno de ellos, aisladamente, ya determinaría la exclusión del territorio en la posible implantación de un uso de los considerados. Sin embargo, hay que tener presente que la acumulación de criterios de restricción modela el resultado de la evaluación final.

Como se ha visto hasta aquí, para componer la estructura de los dos criterios principales de aptitud y vulnerabilidad se han desgranado otros criterios en un proceso de especialización. Estos criterios se configuran en base a una o varias variables territoriales o en base a uno o varios factores. De esta manera, cada alternativa proviene de una variable territorial y con ello cualquier área donde se localice dicha alternativa es en sí misma una posible alternativa. Es cuestión importante enumerar adecuadamente las variables territoriales y los factores que darán lugar a dichos criterios. Ante el considerable número de variables que pudieran surgir, se utilizarán sólo aquellas que se consideran significativas, positiva o negativamente, intentando evitar las redundancias consecuentes de las relaciones existentes entre los elementos y características ambientales. En la figura 1 se muestra la lógica del proceso de evaluación.

Figura 1. Esquema del proceso de evaluación.

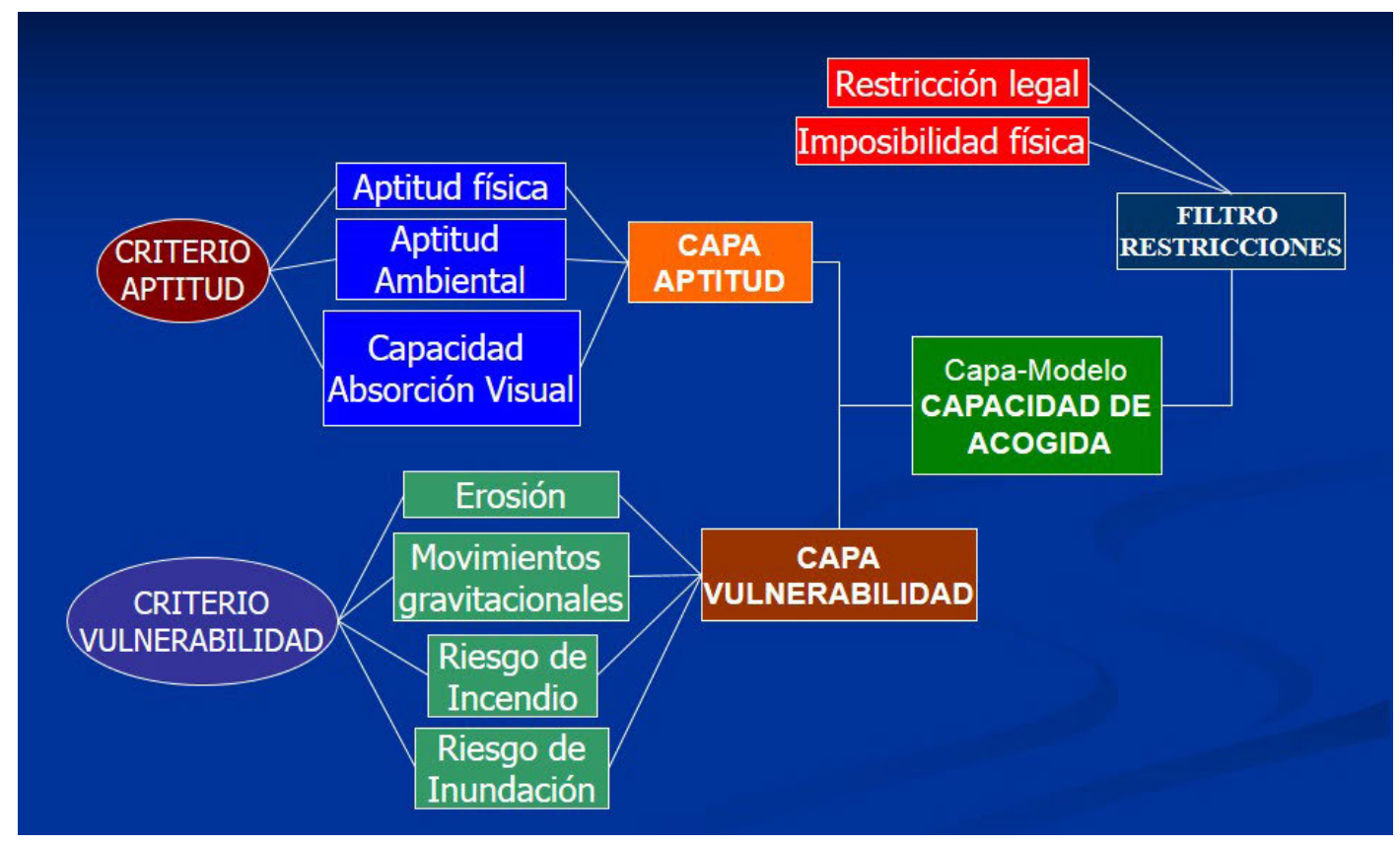

\subsubsection{Puntuación las variables y factores territoriales para su configuración como criterios de la evaluación}

En el apartado anterior hemos definido los criterios de la evaluación y cuál es su composición, es decir, qué variables y factores territoriales los configuran. Ahora se aborda un proceso de puntuación de sus atributos. Si antes proceso de evaluación estaba determinado por las variables territoriales consideradas como se ha comentado, ahora lo está por el tratamiento del que sean objeto dichas variables en su conversión a factores y criterios de evaluación. Los criterios resultantes finalmente albergarán las alternativas existentes, cada alternativa poseerá un conjunto de características que la diferenciarán de otra, y al mismo tiempo, la caracterizarán; así cada alternativa adquirirá una puntuación en función del criterio al que pertenece. 
El proceso que se explica a continuación es realizado para todas las variables. Las variables y factores que dan lugar a los criterios presentarán distintas escalas de medida y además, poseerán en unos casos atributos cuantitativos y en otros cualitativos. Esto supone un problema para la posterior aplicación de las reglas de decisión. Por tanto se trata de operar con los valores de las diferentes variables y factores en una misma escala y expresar sus atributos de forma numérica. Como recurso para realizar esto y representar así la relación de los factores y las alternativas que definen una evaluación es una matriz. En esa matriz, los criterios (j) pueden ocupar la columna principal y las alternativas (i), la fila principal. Dicha matriz la denominaremos matriz de puntuaciones. Ya que los valores internos de esta matriz son llamados puntuaciones de criterios $\left(X_{\mathrm{ij}}\right)$, y representan el valor o nivel de deseabilidad que se establece para cada alternativa en cada factor. Una vez que a una variable o factor se le asignan pesos o puntuaciones este pasa a ser considerado como un criterio.

Para la puntuación de los valores de las variables hemos usado el método de las jerarquías analíticas propuesto por Saaty (1980). Con este procedimiento se establece una matriz cuadrada en cuyas filas y columnas está definido el número de atributos de las variables (clases) a ponderar. El resultado es una matriz de comparación entre pares de clases, en la que se observa la importancia de cada una de ellas sobre cada una de los demás $\left(\mathrm{a}_{\mathrm{ij}}\right)$. La escala de medida establecida para la asignación de los juicios de valor $\left(a_{i j}\right)$ es una escala de tipo continuo (ratios o razón) que va desde un valor mínimo de 1/9 hasta 9 , definida también por Saaty (1980), entendiendo como extremadamente menos importante (1/9) hasta extremadamente más importante (9), indicando el valor 1 de igualdad en la importancia entre pares de factores. Este proceso se presta por su claridad a que sea realizado por técnicos expertos en una materia concreta sin que sea necesario su conocimiento de las técnicas de evaluación multicriterio.

A continuación, en base a las matrices elaboradas, se determina el eigenvector principal, que representa el orden de prioridad de los factores y establece los pesos $\left(\mathrm{w}_{\mathrm{ij}}\right)$, proporcionando una medida cuantitativa de la consistencia de los juicios de valor entre pares de factores como se indica en Saaty (1980). Para cada factor, a partir los juicios de valor entre pares de factores de la matriz de comparación se establece el eigenvector principal, que representará el orden de prioridad de los factores y establecerá los pesos $\left(\mathrm{w}_{\mathrm{ij}}\right)$, pasando a considerar el eigenvalor máximo $\left(\lambda_{M A X}\right)$ de la matriz como una medida de la consistencia de los juicios.

El eigenvector principal conviene que sea normalizado para obtener así el vector de prioridades y que los valores de las alternativas en los distintos ejes estén también normalizados. La normalización en nuestro caso ha sido del tipo $0=$ mínima aptitud -más negativo- y $1=$ máxima aptitud -más positivo-. De los múltiples sistemas de normalización de vectores que pueden utilizarse, se ha optado por uno de los más simples, y se consigue aplicando la siguiente ecuación (Voogd, 1983):

$$
e_{i j}=\frac{\left(x_{i j}-\min x_{i j}\right)}{\left(\max x_{i j}-\min x_{i j}\right)}
$$

Donde:

$e_{i j}$ valor normalizado de la alternativa $i$ en el criterio $j$. $x_{i j}$ valor de la alternativa $i$ en el criterio $j$. min y max: los valores mínimos y máximos de las alternativas en el criterio.

Esta normalización da como resultado valores de 0 a 1 como se ha comentado, con la ventaja de que no efectúa una transformación de la variable, por lo que la proporcionalidad se mantiene. Además, se agradece su utilización cuando el proceso es complejo y nos vemos forzados a volver sobre las puntuaciones y los valores en determinados momentos del proceso de evaluación.

Cuando se trate de las variables cualitativas, la ordenación jerárquica de dichas variables (por ejemplo de mejor a peor) se realiza traduciendo los atributos a valores numéricos, mediante una escala de medida de 1 a 0 o viceversa (operación que se identifica como "función de utilidad"), permite puntuar con un valor numérico a las variables de este tipo. Se advierte que, en este proceder, el problema se desplaza a cómo establecer la ordenación jerárquica, que implica un análisis de preferencias, problema del que tampoco quedaban excluidas las variables cuantitativas, expresadas en escala numérica, cuando no es 
proporcional su preferencia al valor de la variable. En este punto, las preferencias pueden estar claras para el analista (ocurre cuando intervienen juicios técnicos que avalan una determinada jerarquía) o pueden tener un nivel de incertidumbre importante.

\subsubsection{Asignación de pesos a los criterios de la evaluación y aplicación de las reglas de decisión para la consecución de la capa modelo de la evaluación}

Una vez realizadas las tareas descritas en los dos apartados anteriores, los criterios han pasado a formar las partes de un juicio y ser el punto de referencia de las reglas de decisión. Ahora cada criterio será valorado respecto a los demás criterios según las reglas de decisión que se han establecido. Una regla de decisión, en la lógica del proceso de evaluación, se formaliza mediante una serie de procedimientos aritméticos-estadísticos que posibilita la integración de los criterios establecidos en un índice de simple composición, proveyendo la manera de comparar las alternativas utilizando dicho índice (Eastman et al., 1993a). Tales reglas se refieren a aspectos concretos como la medida de los atributos para dar valor a los criterios, o a la forma de integrar los criterios en la evaluación de las alternativas.

Como se sabe, existen dos tipos principales de procedimientos de evaluación multicriterio, desde el punto de vista operativo y de tratamiento de los datos: las técnicas no compensatorias y las técnicas compensatorias (Jankowski, 1995). Las técnicas no compensatorias demandan una jerarquización ordinal de los criterios basada en las prioridades de la evaluación, por tanto mediante la indicación de un valor ordinal o bien el orden de importancia de los criterios, sin establecer un peso de manera cuantitativa. Mientras que las técnicas compensatorias requieren que se especifiquen los pesos de los criterios como valores cardinales o funciones de prioridad, de manera numérica en escala de razón. No nos parece que pueda haber inconveniente en combinar estos dos métodos en el proceso.

En nuestro método, se ha acudido a las técnicas compensatorias, aquellas que manejan simultáneamente los criterios asumiendo la posible compensación entre ellos, en el caso de la evaluación de los criterios de aptitud e vulnerabilidad. En este momento de la evaluación, hemos considerado que los procedimientos compensatorios se adaptan mejor a la lógica del modelo. Dentro de los procedimientos compensatorios, se nos abre la posibilidad de utilizar dos tipos de métodos, como métodos de cálculo para derivar la ordenación lineal de las alternativas a partir de las puntuaciones que se le han adjudicado a los diferentes criterios. Cuando se ha creado un factor desde dos o más variables territoriales se ha utilizado el método aditivo de la sumatoria lineal ponderada y cuando se ha tratado de la configuración de los criterios se ha utilizado la distancia al punto ideal. Creemos que el concepto de situación ideal es fácilmente intuible y la disimilitud respecto a ella es una medida significativa; al mismo tiempo, porque se adapta mejor a la lógica de nuestro modelo de evaluación que aborda un problema de decisión con objetivo simple y permite manejar simultáneamente los criterios asumiendo la posible compensación entre ellos. La opción de la distancia al punto ideal conlleva que el proceso utilice las puntuaciones de las alternativas para medir su similitud con una situación óptima, teórica, que lógicamente estará definida por las mejores puntuaciones posibles en cada criterio. Es una forma de ordenar linealmente las alternativas, también sencilla y clara, en la que lógicamente hay compensación entre los criterios, pero midiendo la desviación de las puntuaciones de las alternativas en cada criterio respecto al valor óptimo y no directamente las propias puntuaciones. A partir de la estructura inicial del procedimiento, podemos establecer que no plantea excesivas limitaciones en su ejecución en relación al número de alternativas a evaluar, ni el número de criterios a ser considerados, lo que le confiere excelentes posibilidades para ser manejado con el SIG. De esta manera, los criterios aptitud física, aptitud ambiental y aptitud funcional que forman el criterio de aptitud y los criterios que forman parte vulnerabilidad al riesgo natural han sido valorados mediante el cálculo de la distancia entre cada alternativa y el punto ideal, de manera que podemos seleccionar aquellas alternativas más cercanas a dicho punto ideal hasta obtener la capa de capacidad de acogida según el objeto de la evaluación sin la aplicación del filtro de restricción, lo que se hará posteriormente (ver figura 1 para ilustración del proceso de evaluación). La ecuación utilizada ha sido la siguiente, tomada de Barredo (1996): 


$$
L p=\left[\sum_{j=1}^{n} w_{j}\left|x_{i j}-1\right|^{p}\right]^{1 / p}
$$

Donde:

$\mathrm{w}_{\mathrm{j}}$ : peso del criterio j. $\mathrm{x}_{i j}$ : valor de la alternativa i en el criterio $\mathrm{j}$; y p: métrica para el cálculo de la distancia ( $\mathrm{p}=2$ corresponde a la distancia euclidiana)

Al resultado anterior, y con finalidad de obtener la capa modelo definitiva hemos aplicado el filtro de restricción. Los criterios de restricción en sí implican, dentro de los juicios que orientan nuestra evaluación, que se trata de limitación absoluta que no puede compensarse con otro factor positivo. Por ello, en este caso, hemos optado por las técnicas no compensatorias. Esta circunstancia tiene su justificación en que no se suelen emplear los criterios de restricción de manera numérica en escala de razón continuas, sino simplemente en forma de presencia o ausencia, puesto que no hay operaciones compensatorias con relación a ellos. Una vez que se han descartado las alternativas donde se aplican los criterios de restricción, el modelo de evaluación barajará combinadamente los criterios de aptitud y de vulnerabilidad para el resto del territorio, es decir, las alternativas posibles.

El resultado de todo el proceso queda sintetizado en una capa de información que muestra una zonificación o clasificación del espacio estudiado con una valoración asignada a cada parte del territorio en función de su capacidad para acoger los usos que han sido evaluados, según se muestra en la figura 2.

Figura 2. Capa modelo resultante del proceso de evaluación.

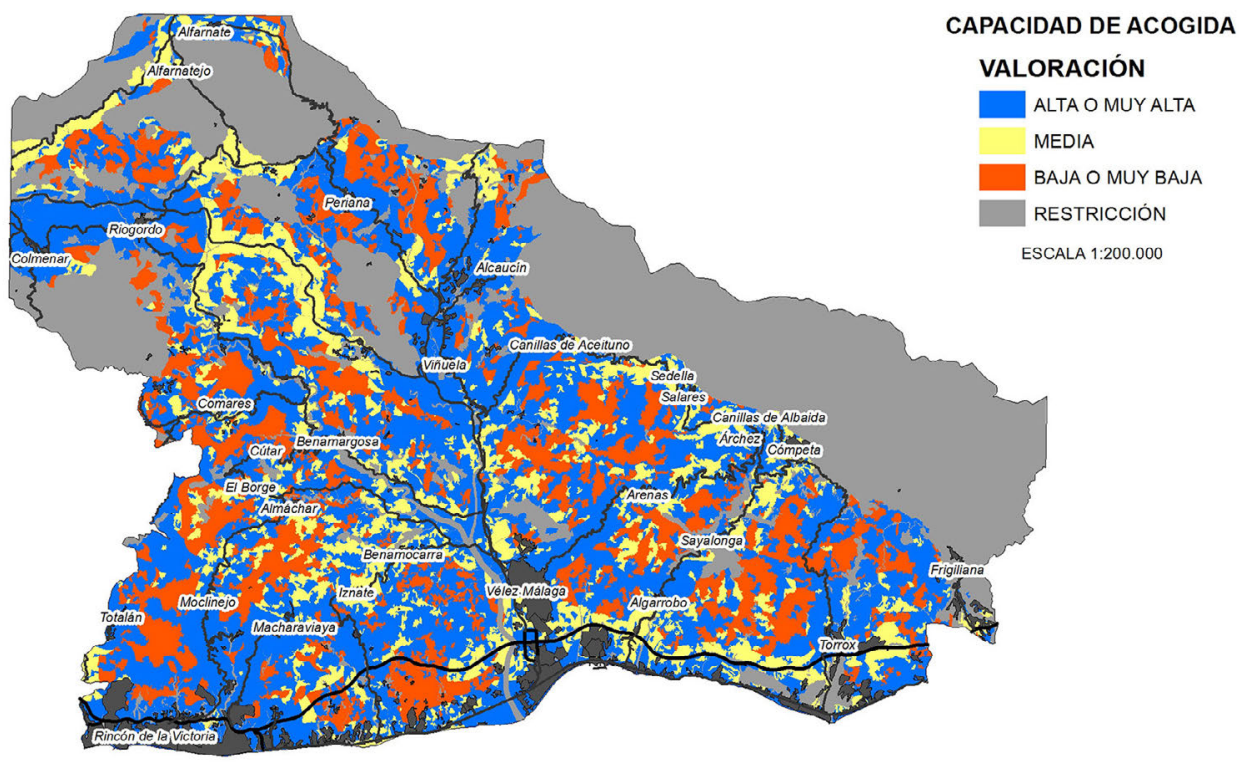

\section{ANÁLISIS DE RESULTADOS}

Una vez obtenida la capa modelo de acogida, analizamos los resultados de su aplicación en un área geográfica concreta de la provincia de Málaga: la comarca de la Axarquía. Este espacio geográfico se localiza en la zona oriental de la provincia de Málaga (España), abarcando una extensión de 1.023,72 km² (12,68 $\%$ de la superficie provincial). Engloba a 31 municipios que pueden ser agrupados en dos realidades territoriales: una, la costa en la que se sitúan 5 municipios que abarcan una superficie de $331,26 \mathrm{~km}^{2}$ $(32,36 \%$ de la superficie de la comarca), y otra, el interior en el que se localizan 26 municipios en una superficie de 692,46 km² (67,64\% de la superficie de la comarca). Sobre este espacio, considerado un área de transición rural-urbana, pero en la que la funcionalidad rural aun es predominante, el proceso urbano, espontáneo y sin planificación, ha irrumpido siguiendo un modelo de urbanismo difuso en suelo rústico. 
El germen de este fenómeno se encuentra en el proceso de ocupación territorial del litoral de la provincia de Málaga ligado a la actividad turística durante las últimas décadas, que ahora se extiende hacia el interior a través de la ocupación indiscriminada del espacio rural bajo una nueva forma de uso residencial turístico; una tipología peculiar de vivienda aislada basada en el diseminado tradicional y en una estructura parcelaria caracterizada por un minifundismo extremo. Los municipios del área aportan el suelo para el desarrollo de este fenómeno y se resarcen así de haber quedado en una posición marginal durante el proceso de desarrollo económico del litoral de las últimas décadas, pasando ahora a ser los protagonistas por su potencialidad residencial ante esta nueva demanda turística: la de la casa en el campo.

En Galacho (2011) se analiza el fenómeno y se muestran unas cifras que tienen como finalidad poner en evidencia la magnitud del fenómeno y algunas de sus características más destacadas en la provincia de Málaga. Hay que hacer notar que el fenómeno reciente de implantación de edificaciones se ha desarrollado, por lo general, de forma irregular cuando no declaradamente ilegal. Aunque también se encuentran casos dentro de la legalidad vigente en el momento de su aparición pero que actualmente podrían enmarcarse como casos en el límite de la arbitrariedad e interpretación abusiva de la normativa urbanística o las leyes del suelo. Aprovechando las circunstancias que prevé la legislación urbanística para la edificación en suelo no urbanizable, y como se ha comentado, apoyadas en las formas de hábitat tradicional en diseminado, se han venido realizando construcciones espontáneas no adscritas a ninguna figura de planeamiento particular (plan parcial, programa de actuación urbanística, proyecto de urbanización, etc.) pero que en muchos casos cuentan con documento administrativo (licencia municipal), es decir, desarrolladas en desacuerdo con el planeamiento pero con permiso municipal.

Las tipologías residenciales de este medio rural son variopintas, y de entre ellas destacamos aquellas relacionadas con el objeto de nuestro estudio: edificaciones de uso residencial ubicados en parcelaciones históricas o en parcelaciones ilegales, formación de núcleos de autoconstrucción por la aglomeración de edificaciones aisladas de modo espontáneo, e implantaciones residenciales de carácter turístico (hoteles rurales y casas rurales fundamentalmente). En conjunto las edificaciones de uso residencial ubicadas en parcelaciones históricas o en parcelaciones ilegales tienen un carácter aislado y se han construido en suelo no urbanizable, la gran mayoría de ellas sin vinculación efectiva con la explotación agrícola o ganadera originaria. Situación que ha venido suponiendo una interpretación abusiva de la legislación vigente en unos casos o un proceso completamente irregular en otros; sobre todo cuando se han producido segregaciones por herencia cuyas partes luego se han vendido por separado, o cuando las parcelaciones históricas han sufrido un proceso de parcelación ilegal. Otra casuística es la derivada de las iniciativas relacionadas con la actividad agraria: naves o almacenes de aperos, que posteriormente pasan a convertirse en viviendas, bien mediante transformación total o bien mediante combinación de ambos usos. En este caso se realizan construcciones espontáneas no adscritas a ninguna figura de planeamiento particular (plan parcial, programa de actuación urbanística, proyecto de urbanización, etc.), carentes de documento administrativo (licencia municipal), o en su caso, si se dispone, es únicamente de licencia municipal para almacén de aperos y objetos de labranza (generalmente se permiten unas instalaciones de una dimensiones muy inferiores de las que realmente se ejecutan).

En este contexto, para completar el análisis hemos realizado un proceso de catalogación del número de edificaciones en el suelo rústico atendiendo fundamentalmente a las tipologías comentadas. Se han contabilizado en la comarca objeto de estudio un total de 22.347 edificaciones en suelo rústico y en situación de irregularidad o ilegalidad. La cuantificación y posterior catalogación se ha realizado por medio de un estudio ortofotogramétrico con SIG y mediante trabajo de campo. La finalidad ahora es evaluar las características de su localización y mediante operaciones de análisis espacial se les ha asignado la valoración contenida en la capa modelo de capacidad de acogida. Con ello establecemos la correlación entre la capacidad de acogida del territorio y la localización de dichas edificaciones e instalaciones. En la figura 3 se muestra el resultado de esta operación. 
Figura 3. Valoración de las edificaciones en relación con la capacidad de acogida.

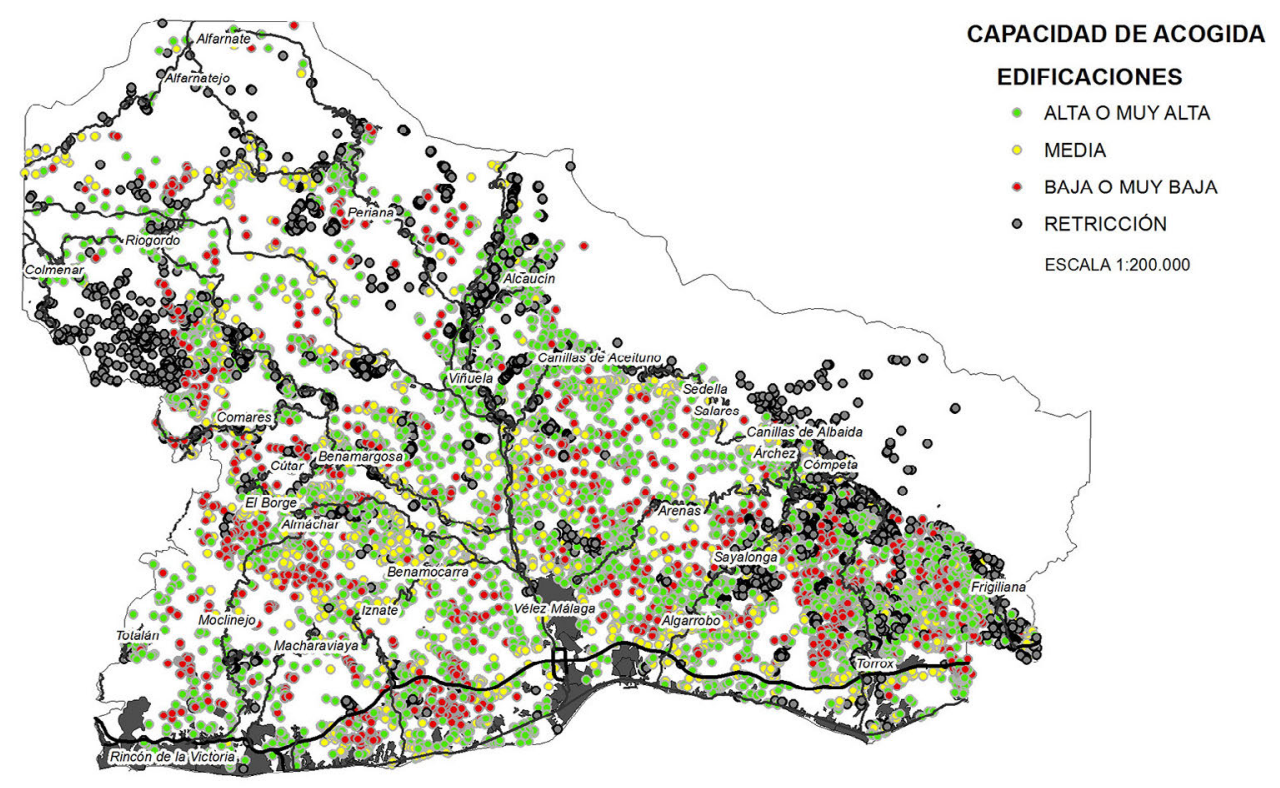

Del total de las edificaciones de uso residencial contabilizadas: 22.347, 2.978 edificaciones se situaban en zonas que han sido valoradas como de capacidad de acogida muy baja o baja ( $13,33 \%$ del total). Por tanto en estas edificaciones se sintetizan unas cualidades territoriales que presentan escasas aptitudes para la construcción, fundamentalmente por la prevalencia de pendientes y tipos litológicos con características que son consideradas no aptas para la ubicación de edificaciones; a su vez, éstas producen una alteración relevante de los valores ambientales de la zona de ubicación por su efecto sobre la vegetación natural y los suelos; y, en su conjunto presentan una escasa integración paisajística, fundamentalmente por tratarse de tipologías no acordes y por el empleo de materiales constructivos ajenos al modelo tradicional. A ello hay que unir que se encuentran en zonas de elevada vulnerabilidad al riesgo natural, fundamentalmente por ser causantes del riesgo de erosión, sobre todo en su fase de construcción y al estar expuestas al riesgo de movimientos gravitacionales (deslizamientos, movimientos en masa y desprendimientos) por las peculiaridades físicas de su ubicación (zonas de elevadas pendientes y situación a media ladera). Con esta valoración suelen coincidir las edificaciones de uso residencial ubicadas en parcelaciones ilegales o diseminados de aparición espontánea.

En zonas que han sido valoradas como de capacidad de acogida intermedia se sitúan 4.904 edificaciones $(21,94 \%$ del total). Estas edificaciones se localizan en zonas en las que confluyen una serie de características físicas y paisajísticas particulares: zonas de relieve alomando y de media altura (entre 400 y 600 metros s.n.m) y pendientes moderada entre el $10 \%$ y el $20 \%$. No obstante, en su evolución reciente hacia un aumento considerable de su número y la formación de conglomerados producen una integración en el medio de difícil encaje, fundamentalmente en lo que se refiere al paisaje. En la mayoría de los casos no se encuentran en zonas de vulnerabilidad al riesgo natural. En este grupo se suelen localizar las edificaciones de uso residencial ubicados en parcelaciones históricas que han sufrido procesos de segregación por herencia e implantaciones residenciales de carácter turístico: hoteles rurales y casas rurales principalmente.

Por otra parte, se han valorado como edificaciones localizadas en zonas de capacidad de acogida muy alta o alta un total de 6.420 edificaciones (28,73\% respecto al total). Esta valoración se corresponde a zonas caracterizadas por una aptitud para la construcción cercana a lo deseable, situadas en las zonas de menor pendiente y relieve más suave, escasamente afectadas por los riesgos considerados, rodeadas de cultivos de subtropicales o frutales lo que favorece su integración paisajística. Este conjunto se corresponde principalmente con los diseminados tradicionales, que si bien en muchos casos tienen otra funcionalidad, han mantenido su localización y morfología originaria, aunque en algunos casos 
hayan sufrido un proceso de restauración y reconversión más o menos acertado. Esto significa que son edificaciones que responden a la lógica territorial del hábitat rural de la zona y que por tanto, surgieron eludiendo aspectos de vulnerabilidad al riesgo natural y buscaron la localización más idónea.

Finalmente, encontramos 8.045 edificaciones (36\% del total) en zonas afectadas por el filtro de restricciones que hemos aplicado: principalmente con prevalencia de las restricciones legales establecidas (dominios públicos, zonas protegidas, servidumbres, vías pecuarias o protecciones impuestas por la planificación urbanística o territorial). En este grupo se encuentran las edificaciones surgidas de modo espontáneo y que han llegado a formar los núcleos de autoconstrucción y las edificaciones aisladas surgidas al margen del planeamiento urbanístico en parcelaciones ilegales, o las surgidas de modo espontáneo por consolidación de edificaciones en antiguas zonas dedicadas a la agricultura y que obtuvieron en su momento una licencia municipal para almacén de aperos y objetos de labranza. Además muchas de estas edificaciones se encuentran en zonas de vulnerabilidad al riesgo de inundación al situarse dentro del dominio público hidráulico.

\section{CONCLUSIONES}

Hemos querido demostrar que las características del proceso de urbanización en el espacio rural engaña en su apariencia, dada su extensión en el territorio y aunque parezca que es menos potente que en los espacios urbanos, en las últimas décadas ha pasado ha extenderse con gran intensidad hacia los espacios que quedaban expeditos de urbanización y hacia los espacios eminentemente rurales interiores. Ante lo constatado parece evidente que un aspecto fundamental que debe ser acometido de modo urgente y regulado a través de las figuras de planeamiento municipal y territorial es el control de las edificaciones y viviendas en el suelo rústico. Aunque este no sea un fenómeno nuevo sino más bien un problema nunca solucionado. De tal manera que es posible afirmar que la introducción en el medio rural del uso residencial no se viene produciendo de modo planificado ni de acuerdo con las condiciones naturales de éste, sino todo lo contrario, la irregularidad de las acciones e implantaciones es la nota predominante.

Con la intención de aportar un instrumento para el diagnóstico y la toma de decisión en el sentido que nos ocupa hemos definido esta metodología cuya lógica es construir un proceso claramente definido cuyo inicio es la formulación del objetivo de la valoración y su fin la obtención de una capa modelo de acogida final. Pero en su lógica interna se pretende no construir un proceso cerrado. Consideramos que con referencia a otras experiencias estudiadas, el modelo avanza en la construcción de la valoración con una desagregación muy elevada de juicios parciales, lo que le convierte en una herramienta dúctil, con la que se puede interactuar, capaz de reorientar la evaluación de acuerdo a diferentes puntos de vista. En este sentido, somos plenamente conscientes que el resultado de la evaluación es valido en función de los juicios y las valoraciones emitidas, teniendo cabida por tanto experimentar sobre resultados alternativos, rectificar los juicios, considerar o no determinadas variables o criterios, etc. Realizado el proceso de evaluación, la información aparece ahora expresada en función del significado del medio en su conjunto para las actividades consideradas.

Teniendo en cuenta que nuestra propuesta metodológica pretende ser dúctil se ha optado por definir ésta en torno a cuatro conceptos básicos: calidad, fragilidad, aptitud y vulnerabilidad al riesgo. Hemos observado como el medio puede ser descrito en función de sus elementos y variables geográficas, y estudiado a través de los conceptos de calidad y fragilidad, la relación de estos dos conceptos con las actividades o usos vendrá dada a través de los conceptos de aptitud y vulnerabilidad al riesgo. Por consiguiente, nos moveremos en un proceso en el que primero se hace una caracterización de las cualidades fisico-ambientales y después una estimación de los distintos tipos de implantaciones. Con ello se ha sinterizado el grado de adaptación de dichas implantaciones al medio, para después se ha hecho una valoración de las alternativas preferidas con la finalidad de cuantificar los efectos negativos derivados de estas acciones. Por lo tanto, se estimarán procedimientos de valoración diferentes según cada caso (definido el caso como el objeto de la evaluación). Es base a la consideración de que las propiedades del territorio tienen un significado en orden al desarrollo de las actuaciones (consideradas estas propiedades en su conjunto, para cada espacio determinado y para unas posibles actuaciones) se define la capacidad que tiene dicho espacio para desarrollar en él dichas actuaciones; así el significado de capacidad de 
acogida del territorio se entiende derivado de la concurrencia, en un espacio determinado, de ciertas características y elementos ambientales significativos. Se trata pues de diseñar procesos de evaluación orientados a objetos específicos con la intensión de delimitar entre las alternativas reales las que sean compatibles con un objeto de evaluación concreto.

Como consecuencia de todo ello se podrán presentar una diversidad de factores susceptibles de intervenir en la definición de los conceptos básicos de aptitud y vulnerabilidad, incluso en el filtro de restricciones; y al mismo tiempo, se configurará un marco de relaciones plural (los lugares de un territorio admiten valoraciones diferentes según la prioridad establecida: el objeto de la evaluación) que sitúa al planificador en el dilema habitual de las alternativas en conflicto.

Un esquema de este tipo permite generar distintas posibilidades de mapas de usos óptimos, por ejemplo, de aptitud o capacidad alta para tipología concretas, pudiendo representar gráficamente qué zonas no responden a un nivel alto de aptitud. Además con este proceso es posible evidenciar también los grados de conflicto que pueden encontrarse y puede darse entrada a gran número de alternativas razonables.

Respecto a esto último y según el número de alternativas barajado, puede ser conveniente realizar una ordenación de las mismas por su capacidad con relación a las cualidades territoriales consideradas de calidad, fragilidad, aptitud y vulnerabilidad. Así se define de forma sistemática una relación de orden $\mathrm{a}_{\mathrm{ej}}>\mathrm{a}_{\mathrm{mi}}$ (la tipología $j$ de la actividad e es preferible a la tipología i de la actividad $m$ ). Esta operación puede resultar laboriosa y es conveniente proceder por aproximaciones sucesivas, entendiendo cada actividad mediante criterios que atiendan al concepto de compatibilidad territorial, tal y como se sugiere en MMA (2000, 669-670) realizar la toma de decisiones según un proceso de análisis aproximativo:

Localización de las tipologías edificatorias sobre la capa modelo resultante del proceso de evaluación y que muestra las clases de aptitud. Se determina en qué áreas sólo una de las tipologías aparece en dos clases superiores. El territorio queda dividido en: áreas a las que se le atribuye ya un uso óptimo (sólo una tipología posible a nivel alto), áreas conflictivas (con más de una tipología posible a nivel alto) y áreas en que ninguna tipología posible alcanza nivel alto.

Relación de orden entre las tipologías que se excluyen o penalizan geográficamente. Se establecen diversas alternativas según relaciones de orden diferentes.

Solución de los conflictos mediante el orden establecido (en las alternativas). Atribución de la tipología que preceda a las demás en el orden de la permutación.

Medición de las superficies resultantes atribuidas a los distintos usos o tipologías de cada uso y toma de decisión de si la distribución es admisible en el contexto general; o si no lo es, se realizan las modificaciones pertinentes en la dirección precisa la relación establecida.

En definitiva, consideramos que es necesario disponer de instrumentos y métodos para una eficaz de ordenación territorial cuyos principios básicos partan de encarar los problemas territoriales mediante la integración de los objetivos económicos, sociales y ambientales. Se aboga por actuar decididamente en la gestión integrada del territorio, mediante la cual se establezcan estrategias de planificación y gestión de los recursos y el espacio. En nuestra consideración, los modelos de capacidad de acogida se adecuan perfectamente a este objetivo, apoyados en las TIGs y las Técnicas de Evaluación Multicriterio. La ideología que rige los planteamientos de esta metodología es que la gestión integrada del territorio implica la voluntad de favorecer que la transformación del espacio rural no se realice bajo los modelos urbanos actuales congestivos y masivos sino que se realice bajo la perspectiva de un sistema más equilibrado apoyado en los núcleos existentes y en los espacios de interés ambiental, cuya integración territorial es fundamental para la consecución de un equilibrio entre el medio rural y el urbano.

\section{BIBLIOGRAFÍA}

BARBA, S. y POMEROL, J. C. (1997): Decisiones multicriterio: fundamentos teóricos y utilización práctica. Servicio de Publicaciones de la Universidad de Alcalá de Henares, Alcalá de Henares, 420 p.

BARREDO, J. I. (1996): Sistemas de información geográfica y evaluación multicriterio en la ordenación del territorio. Ra-Ma Editorial, Madrid, 264 p. 
BARREDO, J. I. y BOSQUE, J. (1995): “Integración de evaluación multicriterio y sistemas de información geográfica para la evaluación de la capacidad de acogida del territorio y asignación de usos del suelo", en Actas IV Congreso Español de Sistemas de Información Geográfica, Barcelona, pp. 191-200.

BELTON, V. y STEWART, T. (2002): Multiple criteria decision analysis: an integrated approach. Kluwer Academic Publishers, Dordrecht, 369 p.

BISSET, R. (1980): "Methods for environmental impact analisys: recent trends and future prospects", en Journal of Environmental Manage, $\mathrm{n}^{\circ} 11$, pp. 27-43.

BLACK, P. E. (1991): Environmental Impact Analisys. Kinko's Center. Syracuse (New York), 310 p.

BOSQUE, J. y MORENO, A. (2004): Sistemas de Información Geográfica y localización de instalaciones y equipamientos. Ra-Ma Editorial, Madrid, 384 p.

CANTER, L. W.:

- (1977, 1996 2a Edición): Environmental Impact Assessment. McGraw Hill. New York, 331 p.

- (1985): Environmental Impacts of Agricultural Production Activities, Lewis Publishers Inc., Chelsea, Michigan, 209 p.

CANTER, L. W. y HILL, L. G. (1979): Handbook of Variables for Environmental Impact Assessment. Ann Arbor. Michigan, 201 p.

CLARK, B. D.:

- (1978): "Methods of environmental impact analysis", en Built Environ, n 4, pp. 111-121.

- (1980): A manual for the assessment of major development proposals. Her Mayesty's Statationery Office. England.

EASTMAN, J. R., KYEM, P. A. y TOLEDANO, J. (1993a): Gis and Decisión Making. United Nations Institute for Training and Research (UNITAR), Ginebra.

EASTMAN, J. R., JIN, W., KYEM, P. A. y TOLEDANO, J. (1993b): “An algorithm for Multi-Objetive Land Allocation Using GIS” en Proceedings International Workshop on GIS, Beijing, Chinese Academy of Science, pp. 261-270.

GAL, T., STEWART, J. y HANNE T. (1999): Multicriteria decision making: advances in MCDM models, algorithms, theory, and applications. Kluwer Academic Publishers, Boston, 560 p.

GALACHO, F. B., OCAÑA, C. y MANCERAS, J. A. (2004): "Diseño de un Sistema de Apoyo a la Decisión Espacial (SADE/SDSS) para la planificación y gestión territorial a escala local", en Actas del XI Congreso de Métodos Cuantitativos, Sistemas de Información Geográfica y Teledetección. Murcia, pp. 13-27

GALACHO, F.B. y OCAÑA, M. C. (2006): "Tratamiento con SIG y Técnicas de Evaluación Multicriterio de la capacidad de acogida del territorio para usos urbanísticos: residenciales y comerciales", Actas del XII Congreso Nacional De Tecnologías de la Información Geográfica, Cd-Rom. Granada, pp. 1.509-1.525.

GALACHO, F.B., RAMÍREZ, J. F., OCAÑA, C., GÓMEZ MORENO, M. L., JUAN, J. I. y ARREBOLA, J. A. (2009): "Desarrollo metodológico para la evaluación de la capacidad de acogida del territorio respecto a actividades ecoturísticas en la cuenca de Valle de Bravo-Amanalco (Estado de México)" en Leyva López, J. C., Avilés Ochoa, E. y Zepeda Rodríguez, J.J. (editores): Herramientas Operativas para el Análisis Multicriterio del Desarrollo Económico Local. Universidad de Occidente, México, pp.165-214.

GALACHO, F.B.:

- (2010): "La irrupción del proceso urbano en los espacios rurales. Reflexiones acerca de las características de una nueva forma de relación entre territorio y economía”, en Delgado, C., Juaristi, J. y Tomé, S. (edit.): Ciudades y paisajes urbanos en el siglo XXI, Ediciones de Librería Estudio. Santander, pp. 297-314.

- (2011): "Implicaciones territoriales y aspectos sociales del urbanismo difuso en áreas de transición rural-urbana. su análisis en la provincia de Málaga (España), en Actas del XXII Congreso de la Asociación de Geógrafos de España, vol. 1. Alicante, pp. 267-278. 
GÓMEZ OREA, D. (1992): Evaluación de Impacto Ambiental. Editorial Agrícola Española S.A. Madrid, 222 p.

GÓMEZ, M. y BARREDO, J. I. (2005): Sistemas de información geográfica y evaluación multicriterio en la ordenación del territorio, Ra-Ma Editorial, Madrid, 279 p.

HOLLICK, M. (1981): "The role of quantitative decision-making methods in environmental impact assessment", en Journal of Environmental Manage, $\mathrm{n}^{\circ}$ 12. pp. 65-78.

JANKOWSKI, P. (1995): "Integrating geographical information systems and multiple criteria decisionmaking methods" en International Journal of Geographical Information Systems, 9, 3, pp. 251-273.

JANSSENS, R. (1992): Multiobjetive decision support for environmental management. Kluwer, Dordrecht, $232 \mathrm{p}$.

LEE, N.:

- (1982): "The future development of environmental impact assessment", en Journal of Environmental Manage, ${ }^{\circ}$ 14. pp. 71-90.

- (1983): "Environmental Impact Assessment: A Review", en Applied Geography, no 3, pp. 60-79.

MALCZEWSKI, J. (1999): GIS and Multicriteria Decision Analisys. John Wiley \& Sons, Inc., New York, $408 \mathrm{p}$.

MMA -Ministerio de Medio Ambiente- (2000): Guía para la elaboración de estudios del medio físico. Centro de Publicaciones de la Secretaria General Técnica del Ministerio de Medio Ambiente, Madrid, 809 p.

MOLERO, E., GRINDLAY, A.L. y ASENSIO, J.J. (2007): "Escenarios de aptitud y modelización cartográfica del crecimiento urbano mediante técnicas de evaluación multicriterio", en GeoFocus, nº 7 , pp. $120-147$.

MORENO, J. M. Y ESCOBAR, M. T. (2000): "El pesar en el proceso analítico jerárquico", en Estudios de Economía Aplicada n ${ }^{\circ} 14$, pp. 95-105.

MORENO, J. M. (2002): "El Proceso Analítico Jerárquico. Fundamentos. Metodología y Aplicaciones", en Caballero, R. y Fernández, G.M.: Toma de decisiones con criterios múltiples. RECT@ Revista Electrónica de Comunicaciones y Trabajos de ASEPUMA, Serie Monografías, nº 1, pp. 21-53.

MORENO JIMÉNEZ, A. Y BUZAI, G.D. (2008): Análisis y planificación de servicios colectivos con Sistemas de Información Geográfica. Departamento de Geografía Universidad Autónoma de Madrid, Madrid, $158 \mathrm{p}$.

MUNDA, G. (2008): Social multi-criteria evaluation for a sustanaible economy. Springer-Verlag, Berlín Heidelberg, $210 \mathrm{p}$.

NIJKAMP, P. y VAN DELFT, A. (1977): Multi-criteria analisys and regional decision making. Martinus Nijhoff. The Netherlands, $135 \mathrm{p}$.

NIJKAMP, P. (1990): Multicriteria evaluation in physical planning. Elsevier Science Publishers, The Nederlands, $219 \mathrm{p}$.

OCAÑA, C. y GALACHO, F.B. (2002): "Un modelo de aplicación de SIG y evaluación multicriterio al análisis de la capacidad de territorio en relación a funciones turísticas", en Actas del IV Congreso Nacional Turismo y Tecnologías de la Información y las Comunicaciones, (Turitec), Málaga, pp. 235-253.

OCAÑA, C., GÓMEZ, Ma.L. y BLANCO, R. (2004): Las vistas como recurso territorial. Ensayo de evaluación del paisaje visual mediante un SIG. Universidad de Málaga (Departamento de Geografía), Málaga, 172 p.

RAU, J. G. y WOOTEN, D. C. (1980): Environmental Impact Analisys Handbook. McGraw Hill. New York, $642 \mathrm{p}$.

ROMERO, C. (1993): Teoría de la decisión multicriterio: conceptos, técnicas y aplicaciones. Alianza Universidad-Textos, Madrid, $195 \mathrm{p}$.

ROY, B. (2007): Metodología multicriterio de ayuda a la decisión. Ediciones Tórculo, Santiago de Compostela, $250 \mathrm{p}$. 
SAATY, T. L. (1980): The Analytical Hierrarchy Process. Mc Graw Hill. Nueva York. 333 p.

SANTOS, J.M.:

- (1997): "El planteamiento teórico multiobjetivo/multicriterio y su aplicación a la resolución de problemas medioambientales y territoriales mediante los SIG raster", en Espacio, Tiempo y Forma (Serie VI, Geografía), no 10 pp. 129-151.

- (1998): "El tratamiento de variables ordinales en la metodología de la evaluación multicriterio (EMC): el método de las precedencias", en Tecnología geográfica para el siglo XXI (Actas del VIII Coloquio del Grupo Métodos Cuantitativos, SIG y Teledetección de la AGE). Barcelona, pp. 120-131.

SEO, F. (1988): Multiple criteria decision analisys in regional planning: concepts, methods and applications. Reidel, London, 230 p.

TRIANTAPHYLLOU, E. (2000): Multi-criteria decision making methods: a comparative study. Kluwer Academic Publishers, Dordrecht, 324 p.

VOOGD, H. (1983): Multicriteria Evaluation for Urban and Regional Planning. Pion, London, 367 p. 